(C) 2012 IEEE. Personal use of this material is permitted. Permission from IEEE must be obtained for all other uses, in any current or future media, including reprinting/republishing this material for advertising or promotional purposes, creating new collective works, for resale or redistribution to servers or lists, or reuse of any copyrighted component of this work in other works. 


\title{
A Discrete Bit Loading Algorithm for FBMC/OQAM
}

\author{
Màrius Caus, Student Member, IEEE, Ana I. Pérez-Neira, Senior Member, IEEE, and \\ Ana García-Armada, Senior Member, IEEE
}

\begin{abstract}
In this letter the discrete rate maximization problem is investigated for FBMC/OQAM. The analysis reveals that if there is crosstalk certain bit allocations violate the power constraints. Aiming at ensuring the feasibility along with alleviating the complexity we have devised a novel iterative algorithm, which always converges. Simulation-based results show that the proposed algorithm performs close to the upper bound for high-coherence bandwidth channels. Under highly frequency selective channels the existing algorithms are not able to guarantee the target SER whereas our approach guarantees the QoS.
\end{abstract}

Index Terms-Discrete rate maximization, FBMC/OQAM.

\section{INTRODUCTION}

M ULTICARRIER modulations (MCM) are widely recognized to be a def nitely powerful technique since the entire band can be equalized with a reduced complexity. In this letter we have focused on a type of MCM namely flter bank based multi carrier (FBMC), which shapes subcarrier signals with frequency localized waveforms. In particular, we have considered the FBMC scheme employing offset quadrature amplitude modulation (FBMC/OQAM) [1]. The author in [2] has shown that FBMC outperforms orthogonal frequency division multiplexing (OFDM) in several areas.

In this letter we delve into the rate maximization (RM) problem imposing the integer-bit constraint. The algorithms that obtain the optimal bit distribution in OFDM, such as [3]-[5], can be directly applied to the FBMC/OQAM case as long as subcarrier signals do neither incur intersymbol interference (ISI) nor intercarrier interference (ICI). Under highly frequency selective channels orthogonality may not be restored so that ICI and ISI terms cannot be neglected. In this scenario the algorithms devised for OFDM lose the optimality, thus

This work was supported by the Spanish Ministry of Science and Innovation under Projects TEC2011-29006-C03-02 (GRE3N-LINK-MAC), TEC2011-29006-C03-03 (GRE3N-SYST), and TEC2008-06327-C03-01, and by the Catalan Government (2009SGR0891). The associate editor coordinating the review of this manuscript and approving it for publication was Prof. Ricardo Merched.

M. Caus is with the Department of Signal Theory and Communications, Universitat Politècnica de Catalunya (UPC), 08034 Barcelona, Spain (e-mail: marius.caus@upc.edu).

A. I. Pérez-Neira is with the Department of Signal Theory and Communications, Universitat Politècnica de Catalunya (UPC), 08034 Barcelona, Spain and also with the Centre Tecnològic de Telecomunicacions de Catalunya (CTTC), 08860 Castelldefels, Barcelona, Spain (e-mail: ana.isabel.perez@upc.edu).

A. García-Armada is with the Department of Signal Theory and Communications, University Carlos III of Madrid, Madrid, Spain (e-mail: agarcia@tsc. uc3m.es). novel techniques have to be designed. Towards this end, the authors in [6] have developed a new formulation for the margin adaptive problem. Benef ting from the work carried out in [6] the main contribution of this letter consists in studying the feasibility of the RM problem in the context of FBMC/OQAM along with highlighting some ideas to reduce the complexity.

The rest of the paper is organized as follows. Section II introduces the system model. In Section III we pose the discrete rate maximization problem. The method to allocate bits is addressed in Section IV. Next, simulation-based results and conclusions are provided in Section V and VI, respectively.

\section{SySTEM MODEL}

This section aims to brief y introduce the system model. At the transmit side, the synthesis flter bank (SFB) output can be expressed as the summation of $M$ subcarrier signals

$$
\begin{aligned}
s[n] & =\sum_{k=-\infty}^{\infty} \sum_{m=0}^{M-1}\left(b_{m}[k]^{*} * x_{m}[k]\right) f_{m}[n-k M / 2] \\
f_{m}[n] & =p[n] \exp \left(j \frac{2 \pi}{M} m(n-(L-1) / 2)\right) .
\end{aligned}
$$

Note that subcarrier signals are shifted versions of the prototype pulse $p[n]$, which length is equal to $L$. Accounting for the transmitted data, let $x_{m}[k]$ be the OQAM symbol transmitted on the $m$-th subcarrier and $k$-th time instant. It is possible to split $x_{m}[k]$ into real PAM symbols $d_{m}[k]$ and a phase term $\theta_{m}[k]$, i.e., $x_{m}[k]=d_{m}[k] \theta_{m}[k]$, where

$$
\theta_{m}[k]=\left\{\begin{array}{lll}
1 & m+k & \text { even } \\
j & m+k & \text { odd }
\end{array}\right\} .
$$

With the objective of confronting multipath fading, subcarrier signals are precoded on a per-subcarrier basis with the multitap linear f lters $\left(b_{0}[k], \ldots, b_{M-1}[k]\right)$. At the receive side, the signal at the analysis f lter bank (AFB) input is given by $r[n]=$ $s[n] * h[n]+w[n]$, where $h[n], w[n]$ account for the channel impulse response and the noise, respectively. According to [1] the symbols are demodulated by implementing a bank of matched $f$ lters and downsampling the outputs with a factor $M / 2$. Hence the signal recovered on the $q$-th band is written in the form

$$
\begin{aligned}
y_{q}[k] & =\sum_{m=q-1}^{q+1}\left(b_{m}[k]^{*} * x_{m}[k]\right) * g_{q m}[k]+w_{q}[k] \\
g_{q m}[k] & =\left(f_{q}[-k]^{*} * h[k] * f_{m}[k]\right)_{\downarrow \frac{M}{2}} .
\end{aligned}
$$

Note that ICI only comes from the adjacent subbands as a consequence of designing $p[n]$ according to [7], with an overlapping factor equal to four. Then, the convolution operations in (4) can be compactly expressed as follows: 


$$
y_{q}[k]=\sum_{m=q-1}^{q+1} \mathbf{b}_{m}^{H} \overline{\mathbf{G}}_{q m}[k] \mathbf{d}_{m}[k]+w_{q}[k] .
$$

For further details we guide the reader to [8]. The last step to compute the symbol estimates consists in de-staggering the AFB output and extracting the real component, that is $\hat{d}_{q}[k]=$ $\Re\left(\theta_{q}[k]^{*} y_{q}[k]\right)$. It is worth mentioning that real PAM symbols can still be formulated in a matrix way if we consider this notation $\mathbf{a}_{e}=\left[\Re\left(\mathbf{a}^{T}\right) \Im\left(\mathbf{a}^{T}\right)\right]^{T}$. Then for the $q+k$ even case $\hat{d}_{q}[k]$ can be expressed as

$$
\hat{d}_{q}[k]=\sum_{m=q-1}^{q+1} \mathbf{b}_{m, e}^{T} \overline{\mathbf{G}}_{q m, e}[k] \mathbf{d}_{m}[k]+\Re\left(w_{q}[k]\right) .
$$

For the sake of brevity we have refrained from writing the expression for the $q+k$ odd case. Without loss of generality transmit vectors are expressed as function of the power allocated on each band, i.e. $\mathbf{b}_{q}=\sqrt{p_{q}} \mathbf{u}_{q}$ where $\left\|\mathbf{u}_{q}\right\|_{2}^{2}=1$. In this letter we have opted to design $\mathbf{u}_{q}$ under the zero forcing (ZF) criterion described in [8] due to its low complexity.

Relying on the fact that the number of interference terms in (7) is suff ciently large, which seems reasonable if the overlapping factor is equal to four, the number of transmittable bits on the $q$-th band can be formulated as follows:

$$
r_{q}=\log _{2}\left(1+\frac{1}{\Gamma} S I N R_{q}\right)
$$

where $\Gamma$ accounts for the signal to noise ratio gap. This term can be approximated to $\Gamma=(1 / 3)\left(Q^{-1}(S E R / 4)\right)^{2}$ as the authors describe in [6]. Along this letter we have considered a symbol error rate (SER) equal to $10^{-4}$. At this point we could formulate the SINR under the assumption that the power allocated on a given band is not constant for consecutive time instants as the authors propose in [6]. The main drawback of this approach is that symbols overlap in the time domain with symbols transmitted on previous and subsequent time instants. Consequently the SINR depends on power coeff cients, which have not been determined yet. This highlights that the problem has to be relaxed. For this reason, we have assumed that channel is quasi-static. As a result, the power allocated on a given band remains constant for consecutive time instants. The proposed simplif cation yields the following SINR

$$
\begin{aligned}
\operatorname{SINR}_{q}= & \frac{p_{q}\left\|\mathbf{u}_{q, e}^{T} \overline{\mathbf{G}}_{q q, e}[k] \mathbf{e}_{l}\right\|_{2}^{2}}{i_{q}+N_{0} / 2}=\frac{p_{q} h_{q}}{i_{q}+N_{0} / 2} \\
i_{q}= & p_{q-1}\left\|\mathbf{u}_{q-1, e}^{T} \overline{\mathbf{G}}_{q q-1, e}[k]\right\|_{2}^{2} \\
& +p_{q+1}\left\|\mathbf{u}_{q+1, e}^{T} \overline{\mathbf{G}}_{q q+1, e}[k]\right\|_{2}^{2} \\
& +p_{q}\left\|\mathbf{u}_{q, e}^{T} \overline{\mathbf{G}}_{q q, e}[k]\left(\mathbf{I}-\mathbf{e}_{l} \mathbf{e}_{l}^{T}\right)\right\|_{2}^{2} \\
i_{q}= & p_{q-1} \alpha_{q q-1}+p_{q+1} \alpha_{q q+1}+p_{q} \alpha_{q q} .
\end{aligned}
$$

Let $\alpha_{q m}$ be the interference that comes from the $m$-th subband and $N_{0}$ be the power spectral density of the noise. Since we extract the real dimension of the samples, the power of the noise is halved. The vector $\mathbf{e}_{l}$, which is zero-valued except in the $l$ th position, selects the desired stream. It can be verif ed that (9), (10) are also valid for the $q+k$ odd case.

\section{Problem Statement And FEASIBILITy ANALysis}

This section poses the RM problem as well as determines its feasibility. For practicality reasons we have limited the rates to be integer, which leads to the following problem:

$$
\begin{aligned}
& P: \max _{p_{q}} \sum_{q=0}^{M-1} r_{q} \\
& \text { s.t. } \sum_{q=0}^{M-1} p_{q} \leq P_{T}, p_{q} \geq 0, r_{q} \in\left\{0,1, \ldots, N_{q}\right\} .
\end{aligned}
$$

Let $N_{q}$ be the maximum number of transmittable bits on the $q$-th band, which depends on the value at which $r_{q}$ saturates. The bound is def ned as $N_{q}=\left\lfloor\log _{2}\left(1+h_{q} /\left(\alpha_{q q} \Gamma\right)\right)\right\rfloor$. Similarly to [6] our approach to solve (12) is based on a bit-f lling algorithm. The idea is to increase the rate in the band that exhibits the least incremental power to convey an aditional bit. However, the formulation is different since we have made no assumption about the $f$ atness of the channel at each subcarrier. In this sense, provided that on the $j$ th iteration the number of bits loaded so far on the $q$ th band is denoted $r_{q}(j)$, the power required on the $q$ th band is computed from (8) and is given by

$$
p_{q}^{j}=\frac{\Gamma\left(2^{r_{q}(j)}-1\right)\left(N_{0} / 2+p_{q-1}^{j} \alpha_{q q-1}+p_{q+1}^{j} \alpha_{q q+1}\right)}{h_{q}-\alpha_{q q} \Gamma\left(2^{r_{q}(j)}-1\right)} .
$$

From (13) it can be inferred that in the next iteration, the additional power required to transmit one more bit on the $q$ th band, i.e., $\Delta_{q}^{j+1}=p_{q}^{j+1}-p_{q}^{j}$, reads as follows:

$$
\Delta_{q}^{j+1}=\frac{\Gamma h_{q} 2^{r_{q}(j)}\left(N_{0} / 2+p_{q-1}^{j} \alpha_{q q-1}+p_{q+1}^{j} \alpha_{q q+1}\right)}{\left(h_{q}-\alpha_{q q} \Gamma\left(2^{r_{q}(j)}-1\right)\right)\left(h_{q}-\alpha_{q q} \Gamma\left(2^{r_{q}(j)+1}-1\right)\right)} .
$$

It is worth emphasizing that (8) is monotonically increasing in $p_{q}$ and monotonically decreasing in $p_{q-1}$ and $p_{q+1}$. This highlights that the vector of powers $\mathbf{p}(j)=\left[p_{0}^{j}, \ldots, p_{M-1}^{j}\right]^{T}$ cannot be updated on a per-subcarrier basis. Taking into consideration (13) we can use the following matrix notation to compactly formulate the relation between the power coeff cients and the bits loaded at each iteration

$$
\begin{aligned}
& \left(\mathbf{I}_{M}-\mathbf{A}(j)\right) \mathbf{p}(j)=\mathbf{w}(j) \\
& \mathbf{w}(j)=\frac{N_{0}}{2}\left[\frac{\Gamma\left(2^{r_{0}(j)}-1\right)}{h_{0}}, \ldots, \frac{\Gamma\left(2^{r_{M-1}(j)}-1\right)}{h_{M-1}}\right]^{T}
\end{aligned}
$$

where $\mathbf{I}_{M}$ is the identity matrix and $\mathbf{A}(j)$ is an $M \times M$ matrix, which accommodates ICI and ISI. For $l=0, \ldots, M-1$ the matrix is def ned as follows

$$
[\mathbf{A}(j)]_{l t}=\left\{\begin{array}{ll}
\alpha_{l t} \frac{\Gamma\left(2^{r_{l}(j)}-1\right)}{h_{l}}, & t \in\left\{l_{-1}, l, l_{1}\right\} \\
0, & \text { otherwise }
\end{array}\right\}
$$

where the operation $l_{a}=(l+a)_{\bmod M}$ computes the modulus $M$ of the argument. Note that it is mandatory to compute $\mathbf{C}(j)=$ $\left(\mathbf{I}_{M}-\mathbf{A}(j)\right)^{-1}$ to recalculate the powers. The complexity is reduced when the matrix inversion lemma comes into action. Provided that at the $j$ th iteration an extra bit is loaded on the $l$ th subband, the matrix $\mathbf{C}(j)=\left(\mathbf{I}_{M}-\mathbf{A}(j-1)+\mathbf{v}_{l} \mathbf{z}_{l}^{T}(j-1)\right)^{-1}$ is given by 


$$
\begin{aligned}
& \mathbf{C}(j)=\mathbf{D}(j-1) \mathbf{C}(j-1) \\
& \mathbf{C}(j)=\left(\mathbf{I}_{M}-\frac{\mathbf{C}(j-1) \mathbf{v}_{l} \mathbf{z}_{l}^{T}(j-1)}{1+\mathbf{z}_{l}^{T}(j-1) \mathbf{C}(j-1) \mathbf{v}_{l}}\right) \mathbf{C}(j-1)
\end{aligned}
$$

where $\mathbf{v}_{l}$ is a column vector, which takes the value one at the $l$ th position whereas it is zero-valued in the rest of positions. The elements collected in the vector $\mathbf{z}_{l}(j-1)$, which simplif es the update of $\mathbf{C}(j)$, are expressed as follows:

$$
\left[\mathbf{z}_{l}(j-1)\right]_{t}=\left\{\begin{array}{ll}
-\alpha_{l t} \frac{\Gamma 2^{r_{l}(j-1)}}{h_{l}} & t \in\left\{l_{-1}, l, l_{1}\right\} \\
0 & \text { otherwise }
\end{array}\right\} .
$$

When updating the power it is crucial that the algorithm makes sure that $\mathbf{p}(j)$ is element-wise positive. From the theory developed in [9], we know that the rates on the $j$ th iteration are achievable if and only if the spectral radius of $\mathbf{A}(j)$ is lower than one. Since the complexity of carrying out a singular value decomposition at each iteration would be unaffordabale we can circumvent the problem under the assumption that $\mathbf{C}(j-1)$ is element-wise positive. In other words, the bits loaded in the last step should be achievable. Initializing the algorithm with $\mathbf{C}(0)=\mathbf{I}_{M}$, the condition

$$
\mathbf{z}_{l}^{T}(j-1) \mathbf{C}(j-1) \mathbf{v}_{l} \geq-1
$$

is suff cient to ensure that the new sum-rate after allocating an extra bit on the $l$ th subband does not violate any power constraint. If the inequality (20) is not satisf ed, then $\mathbf{D}(j-1)$ is element-wise negative out of the diagonal. Hence $\mathbf{p}(j)$ is not positive if just a single element in the diagonal of $\mathbf{D}(j-1)$ is negative. To sum up, when (20) does not hold we cannot state that $\mathbf{p}(j) \geq 0$ if

$$
1-\frac{\min _{i=0, \ldots, M-1}\left(\left[\mathbf{C}(j-1) \mathbf{v}_{l} \mathbf{z}_{l}^{T}(j-1)\right]_{i i}\right)}{1+\mathbf{z}_{l}^{T}(j-1) \mathbf{C}(j-1) \mathbf{v}_{l}} \leq 0 .
$$

In the third and last case, where (20) and (21) are not fulf lled, the vector $\mathbf{p}(j)$ may be element-wise positive while some positions of $\mathbf{C}(j)$ may contain negative elements. If so, (20) does not guarante the positivity of $\mathbf{p}(j)$, thus the feasibility on subsequent iterations would hinge on checking the spectral radius of $\mathbf{A}(j)$. With the aim of reducing the complexity burden when (20) does not hold we assume that at least one element in the diagonal of $\mathbf{D}(j-1)$ is negative, which implies that the sum-rate is not achievable and as a result the $l$ th band is discarded.

\section{Bit AND Power Allocation Algorithm}

It can be readily verif ed that the proposed bit-f lling algorithm converges faster to the solution if the initial bit and power distributions are different from zero. In this regard, we exploit the good spectral conf nement of the pulses to set the initial bit and power prof les. The idea consists in collecting the indexes of the bands in two subsets so that subcarrier signals belonging to the same subset do not overlap in the frequency domain. This is achieved by taking one subcarrier out of two, which results in these two subsets: $\mathrm{S} 1=\{0,2, \ldots, M-2\}$ and $\mathrm{S} 2=\{1,3, \ldots, M-1\}$. Consequently, if we f rst focus
TABLE I

Pseudocode Implementing Bit LoAding AND Power Allocation

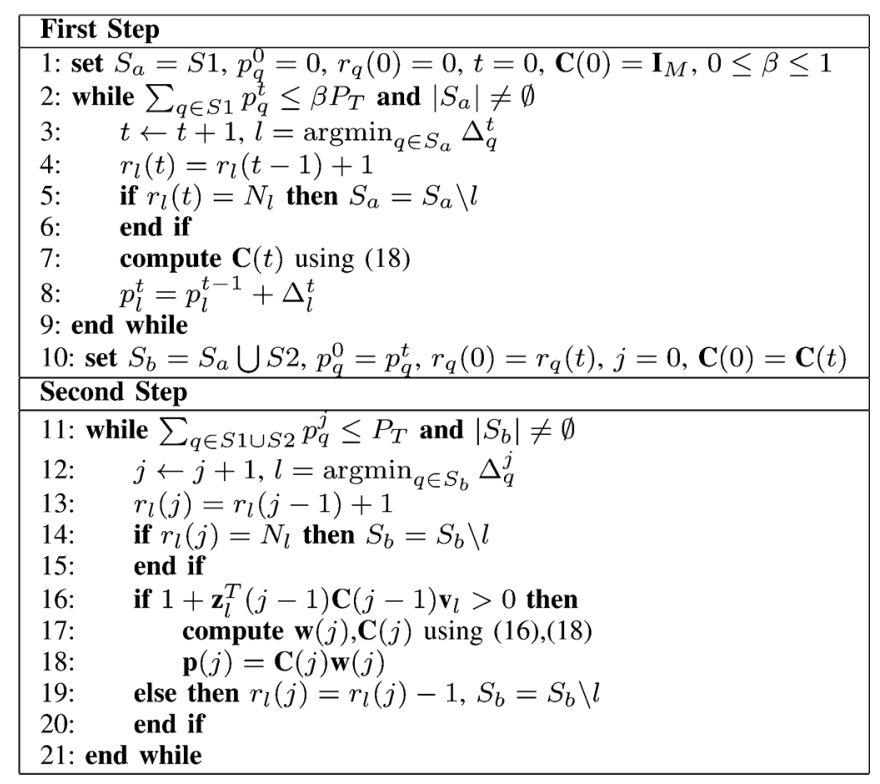

on S1, the bands can be loaded with bits with a reduced complexity. The key aspect is that the f nal bit prof le on the subset $\mathrm{S} 1$ can be regarded as the initial bit distribution. Bearing this in mind we have divided the algorithm in two parts, which are described in Table I. In the frst step we assign one more bit to the least demanding band, which belongs to S1, in terms of incremental power. Note that the proposed algorithm also partitions the power budget by means of the parameter $\beta$. Subsequently, in the second step all the bands come into play. Note that the parameters $\mathbf{C}(t), p_{q}^{t}, r_{q}(t)$ and $\Delta_{q}^{t}$ computed in the last iteration of the frst step are fed to the second part and are used to initialize the algorithm. It is mandatory that in the second step subsets S1 and S2 have to be jointly addressed. Otherwise the SINR of those bands addressed in the f rst step would degrade, thus the target SER would not be guaranteed. It is likely that if we increase $\beta$, the bands with low channel gains are assigned bits to the detriment of bands belonging to S2, which may be more eff cient. However, by breaking the algorithm in two parts the complexity in the frst step is reduced. As a result, the additional complexity with respect to the bit-f lling algorithm applied to OFDM is bounded by $\mathcal{O}\left(I M^{2}\right)$, where $I$ is the number of iterations performed in the second step and $M$ is the number of carriers.

It is important to remark that the condition in line 16 ensures that $\mathbf{p}(j) \geq 0$, which guarantess the convergence of the algorithm since there is a total power constraint.

\section{Simulation Results}

This section evaluates the sum-rate in two different scenarios. In the f rst one the number of carriers and the subcarrier spacing are set to $M=512$ and $\Delta f=10.94 \mathrm{KHz}$, respectively. By contrast, in the second case we have f xed $M=256$ and $\Delta f=$ $21.88 \mathrm{KHz}$. As for the propagation conditions the ITU-Vehicular A and ITU-Vehicular B channel models have been adopted in the f rst and second scenario, respectively. As a consequence, 


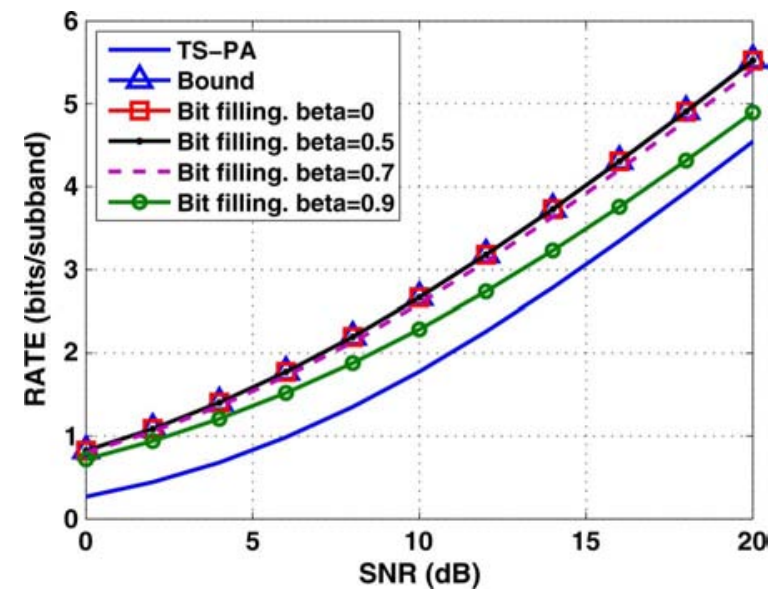

Fig. 1. Rate versus SNR. Scenario 1.

subchannels are $\mathrm{f}$ at fading in the $\mathrm{f}$ rst case whereas there is appreciable frequency selectivity in the second one.

We have considered the technique of [6] as a benchmark to compare with. In particular, we have implemented the power allocation of [6] in the second step having set the initial bits and powers to zero. It is important to highlight that the ISI and ICI terms derived in [6] are modeled under the assumption that the channel is $f$ at at each subcarrier. This implies that the interference depends on the prototype pulse since precoders perfectly equalize the channel. The waveform used in this letter fulf ls the nearly perfect reconstruction property, thus the residual interference is negligible when the channel is $f$ at fading at each subcarrier. In this scenario the incremental power to convey one more bit, to any given band, is the same when compared with OFDM. This allows us to conclude that the benchmark converges to the same solution as the greedy algorithm used in OFDM, which is identif ed as 'bound'. However, if the interferences are not negligible the SINR degrades, thus the target SER is not preserved. In order to prevent this from happening we should recalculate the transmittable rates disregarding the ideal SINR, which is calculated under the $\mathrm{f}$ at fading assumption, in favour of the real SINR. In addition, a f nal rounding step should be performed to ensure that bits are integer. This technique will be called two-step power allocation (TS-PA) henceforth.

In Fig. 1 and Fig. 2 the average rate $\left(\sum_{q=0}^{M-1} r_{q} / M\right)$ is plotted against the signal to noise ratio (SNR) def ned as $\left(P_{T} /\left(M N_{0}\right)\right)$. Under the conditions simulated in Fig. 1 the proposed solution with $\beta=0$ coincides with the bound whereas outperforms the TS-PA in one bit per carrier. For SNR $=20 \mathrm{~dB}$ the ideal SINR, used by the bound, is on average $0.017 \mathrm{~dB}$ away from the real SINR. Therefore the effect of neglecting the interferences has very little impact. Regarding the addressed algorithm, Fig. 1 shows that we can allocate up to $70 \%$ of the power in the frst step without substantially reducing the rate.

Note that in Fig. 2 the bound has not been depicted because the gap between the real and the ideal SINR is on average $2 \mathrm{~dB}$. This difference leads to a loose bound, which is not meaningful. It must be mentioned that in scenario 2 the $\mathrm{CP}$ length required by OFDM should be larger than $M / 4$ samples. As for the rest of techniques, the relative behaviour between them is very similar

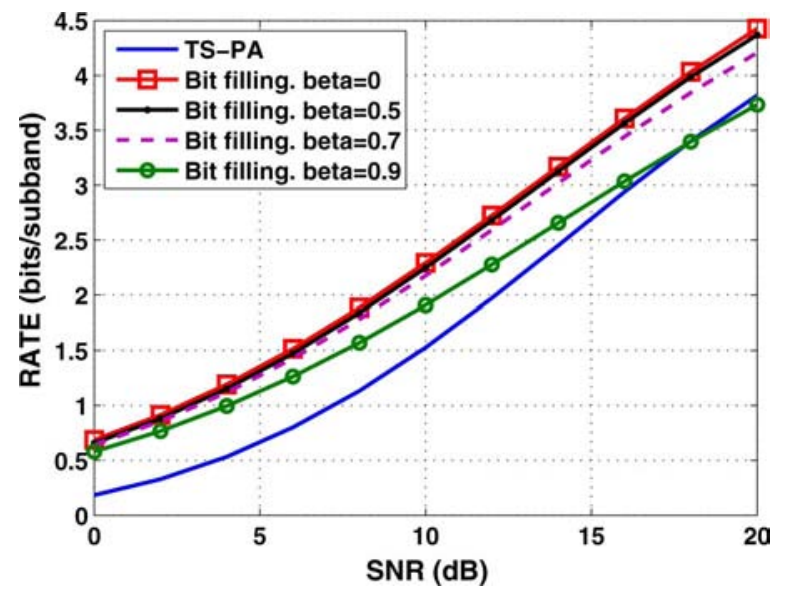

Fig. 2. Rate versus SNR. Scenario 2.

when compared to Fig. 1. It is worth mentioning that we have encountered several cases of unfeasibility under the conditions simulated in Fig. 2. Nonetheless, we have empirically observed that whenever (20) does not hold (21) is always satisf ed, which conf rms the validity of our approach to ensure the convergence.

As $\beta$ increases the number of iterations performed in the second step reduces albeit the sum-rate degrades. In this sense, the results show that the best tradeoff is achieved by $\beta=0.7$.

\section{CONCLUSIONS}

In this letter, we have tackled the discrete RM problem for FBMC modulations. The analysis concludes that some bit distributions should be prohibited otherwise the algorithms based on a bit flling approach diverge. Aiming at avoiding this situation we have devised a novel suboptimal approach, which ensures the convergence. The proposed algorithm has been shown to provide the same results as the upper bound when the channel frequency response is $f$ at at each subcarrier. For more severe propagation conditions the existing solutions, unlike the proposed algorithm, do not preserve the target SER.

\section{REFERENCES}

[1] P. Siohan, C. Siclet, and N. Lacaille, "Analysis and design of OFDM/OQAM systems based on flterbank theory," IEEE Trans. Signal Process., vol. 50, no. 5, pp. 1170-1183, May 2002.

[2] B. Farhang-Boroujeny, "OFDM versus f lter bank multicarrier," IEEE Signal Process. Mag., vol. 28, no. 3, pp. 92-112, May 2011.

[3] D. Hughes-Hartogs, "Ensemble Modem Structure for Imperfect Transmission Media," U.S. Patent 4,679,227, Jul. 1987.

[4] J. Campello, "Practical bit loading for DMT," in ICC, 1999, vol. 2, pp. $801-805$.

[5] R. Sonalkar and R. Shively, "An eff cient bit-loading algorithm for DMT applications," IEEE Commun. Lett., vol. 4, no. 3, pp. 80-82, Mar. 2000.

[6] K. El Baamrani, V. Jimenez, A. Armada, and A. Ouahman, "Multiuser subcarrier and power allocation algorithm for OFDM/Offset-QAM," IEEE Signal Process. Lett., vol. 17, no. 2, pp. 161-164, Feb. 2010.

[7] M. Bellanger, "Specif cation and design of a prototype flter for flter bank based multicarrier transmission," in ICASSP, 2001, pp. 2417-2420.

[8] M. Caus and A. I. Perez-Neira, "Transmit and receive f lters for MISO FBMC systems subjected to power constraints," in ICASSP, May 2011, pp. $2660-2663$.

[9] H. Boche and M. Schubert, "A general duality theory for uplink and downlink beamforming," in Proc. VTC, Fall, 2002, vol. 1, pp. 87-91. 